\title{
Existence of solutions to strongly damped quasilinear wave equations
}

\author{
Hong Luo ${ }^{1 *}$, Li-mei Li and Tian Ma²
}

${ }^{*}$ Correspondence:

Ihscnu@hotmail.com

${ }^{1}$ College of Mathematics and

Software Science, Sichuan Normal

University, Chengdu, Sichuan

610066, China

Full list of author information is

available at the end of the article

\begin{abstract}
In this paper, we study the strongly damped quasilinear wave equation. By using spatial sequence techniques and energy estimate methods, we obtain the existence theorem of the solution to abstract a strongly damped wave equation and to a class of strongly damped quasilinear wave equations.
\end{abstract}

MSC: 35L05; 35L20; 35D30; 35D35

Keywords: existence; solution; wave equations; strongly damped; quasi-linear

\section{Introduction}

This paper is concerned with the following initial-boundary problem of strongly damped quasilinear wave equations:

$$
\left\{\begin{array}{l}
\frac{\partial^{2} u}{\partial t^{2}}-k \frac{\partial \Delta u}{\partial t}=-\sum_{|\alpha| \leq m}(-1)^{|\alpha|} D^{\alpha} A_{\alpha}\left(x, u, \ldots, D^{m} u\right)+g\left(x, u, \ldots, D^{m} u\right) \\
\left.u\right|_{\partial \Omega}=\cdots=\left.D^{m-1} u\right|_{\partial \Omega}=0 \\
u(x, 0)=\varphi, \quad u_{t}(x, 0)=\psi
\end{array}\right.
$$

where $k>0, m \geq 1, \Delta$ is the Laplacian operator, $\Omega$ denotes an open bounded set of $R^{n}$ with smooth boundary $\partial \Omega$, and $u$ denotes vertical displacement at $(x, t)$.

Equation (1.1) is a quasilinear wave equation with strong damping, which has many applications. The existence and asymptotic behavior for the strongly damped wave equations have been extensively studied by many authors [1-15]. Local well-posedness for strongly damped wave equations with critical nonlinearities is studied in [2]. The existence and asymptotic behavior for a strongly damped nonlinear wave equation have been concerned in $[1,3-9,12-15]$. Fan [10] investigated the existence and the continuity of the inflated attractors for a class of nonautonomous strongly damped wave equations through differential inclusion. Li [11] obtained the existence of a global periodic attractor attracting any bounded set exponentially in the phase space by introducing a new norm, which is equivalent to the usual norm.

The quasilinear wave equation has been investigated by many authors in the last years [16-28]. In [16-20], it is considered the boundary value problem for the quasilinear wave equation. Under certain assumptions, the global smooth solvability is obtained. It has been shown by Alinhac $[21,22]$ that the null condition implies global existence of smooth solutions in two space dimensions. Zhang [23] studies the global existence, singularities, 
and life span of smooth solutions of the Cauchy problem for a class of quasilinear hyperbolic systems with higher order dissipative terms and gives their applications to nonlinear wave equations with higher order dissipative terms. Metcalfe and Sogge [24] give a simple proof of global existence for quadratic quasilinear Dirichlet-wave equations outside of a wide class of compact obstacles in the critical case where the spatial dimension is three. Yin [25] gives the lower bound of a lifespan of classical solutions and discusses the long time asymptotic behavior of solutions away from the blowup time. Weidemaier [26] establishes local (in time) existence of classical solutions to the initial-boundary value problem for a quasilinear wave equation. In [27], the existence and uniqueness of the classical solutions for the initial value problems and the first boundary problems of a quasilinear wave equation are proved by the Galerkin method. In [28], the numerical solution for a type of quasilinear wave equation is studied. The three-level difference scheme for quasilinear waver equation with strong dissipative term is constructed and the convergence is proved.

The strongly damped wave equations and the quasilinear wave equation have a lot of results. But up to now, we find several results on the strongly damped quasilinear wave equation. Chen [29] shows that the initial boundary value problem for the strongly damped quasilinear wave equation has a global solution and that there exists a compact global attractor with finite dimension. Comparing Eq. (1.1) and [29], we find that $A_{\alpha}\left(x, u, \ldots, D^{m} u\right)=\sigma\left(u_{x}\right)_{x}, g\left(x, u, \ldots, D^{m} u\right)=-f(u)+g(x)$, and $x \in \Omega=[0,1]$. In this article, our interest is to study that Eq. (1.1) has a solution under which condition of $A$ and $g$. This article uses the spatial sequence techniques, each side of the equation to be treated in different spaces, which is an important way to get more extensive and wonderful results.

The outline of the paper is as follows. In Section 2, we provide essential preliminaries, which include definitions and lemmas from [30]. In Section 3, we give existence of solutions to abstract strongly damped wave equations. In Section 4, we present the main results and their proof. Existence of solutions to a class of strongly damped quasilinear wave equations is given.

\section{Preliminaries}

We introduce two spatial sequences:

$$
\left\{\begin{array}{l}
X \subset H_{3} \subset X_{2} \subset X_{1} \subset H \\
X_{2} \subset H_{2} \subset H_{1} \subset H
\end{array}\right.
$$

where $H, H_{1}, H_{2}, H_{3}$ are Hilbert spaces, $X$ is a linear space, and $X_{1}, X_{2}$ are Banach spaces. All imbeddings of (2.1) are dense. Let

$$
\left\{\begin{array}{l}
L: X \rightarrow X_{1} \quad \text { is one-one dense linear operator, } \\
\langle L u, v\rangle_{H}=\langle u, v\rangle_{H_{1}}, \quad \forall u, v \in X
\end{array}\right.
$$

Furthermore, $L$ has eigenvectors $\left\{e_{k}\right\}$ satisfying

$$
L e_{k}=\lambda_{k} e_{k} \quad(k=1,2, \ldots)
$$

and $\left\{e_{k}\right\}$ constitutes a common orthogonal basis of $H$ and $H_{3}$. 
We consider the following abstract equation:

$$
\left\{\begin{array}{l}
\frac{d^{2} u}{d t^{2}}+k \frac{d}{d t} \mathcal{L} u=G(u), \quad k>0 \\
u(0)=\varphi, \quad u_{t}(0)=\psi
\end{array}\right.
$$

where $G: X_{2} \times R^{+} \rightarrow X_{1}^{*}$ is a map, $R^{+}=[0, \infty)$ and $\mathcal{L}: X_{2} \rightarrow X_{1}$ is a bounded linear operator satisfying

$$
\langle\mathcal{L} u, L v\rangle_{H}=\langle u, v\rangle_{H_{2}}, \quad \forall u, v \in X_{2}
$$

Definition 2.1 We say $u \in W^{1, \infty}\left((0, T), H_{1}\right) \cap L^{\infty}\left((0, T), X_{2}\right)$ is a global weak solution of Eq. (2.4) provided that $(\varphi, \psi) \in X_{2} \times H_{1}$

$$
\left\langle u_{t}, v\right\rangle_{H}+k\langle\mathcal{L} u, v\rangle_{H}=\int_{0}^{t}\langle G(u), v\rangle d t+\langle\psi, v\rangle_{H}+k\langle\mathcal{L} \varphi, v\rangle_{H}
$$

for all $v \in X_{1}$ and $0 \leq t \leq T<\infty$.

Definition 2.2 Let $u_{n}, u_{0} \in L^{p}\left((0, T), X_{2}\right)$. We say $u_{n} \rightarrow u_{0}$ in $L^{p}\left((0, T), X_{2}\right)$ is uniformly weakly convergent if $\left\{u_{n}\right\} \subset L^{p}((0, T), H)$ is bounded, and

$$
\left\{\begin{array}{l}
u_{n} \rightarrow u_{0} \quad \text { in } L^{p}\left((0, T), X_{2}\right), \\
\lim _{n \rightarrow \infty} \int_{0}^{T}\left|\left\langle u_{n}-u_{0}, v\right\rangle_{H}\right|^{2} d t=0, \quad \forall v \in H .
\end{array}\right.
$$

Definition 2.3 We say that a map $G: X_{2} \times(0, \infty) \rightarrow X_{1}^{*}$ is $T$-coercive weakly continuous if for all $\left\{u_{n}\right\} \subset L_{\mathrm{loc}}^{p}\left((0, \infty), X_{2}\right) \cap L_{\mathrm{loc}}^{\infty}((0, \infty), H), u_{n} \rightarrow u_{0}$ in $L^{p}\left((0, T), X_{2}\right)$ is uniformly weakly convergent, and

$$
\lim _{n \rightarrow \infty} \int_{0}^{t}\left|\left\langle G u_{n}-G u_{0}, L u_{n}-L u_{0}\right\rangle\right| d t=0, \quad 0<T<\infty
$$

then

$$
\lim _{n \rightarrow \infty} \int_{0}^{t}\left|\left\langle G u_{n}, v\right\rangle\right| d t=\lim _{n \rightarrow \infty} \int_{0}^{T}\left|\left\langle G u_{0}, v\right\rangle\right| d t, \quad \forall v \in X_{1}, 0<t<\infty .
$$

Lemma 2.4 ([30]) Let $\left\{u_{n}\right\} \in L^{p}\left((0, T), W^{m, p}(\Omega)\right)(m \geq 1)$ be bounded sequences, and $\left\{u_{n}\right\}$ uniformly weakly convergent to $\left\{u_{0}\right\} \in L^{p}\left((0, T), W^{m, p}(\Omega)\right)$. Then, for all $|\alpha| \leq m-1$, it follows that

$$
D^{\alpha} u_{n} \rightarrow D^{\alpha} u_{0} \quad \text { in } L^{2}((0, T) \times \Omega)
$$

Lemma 2.5 ([30]) Let $\Omega \subset R^{n}$ be a open set, and $: \Omega \times R^{N} \rightarrow R^{1}$ satisfy the Caratheodory condition and

$$
|f(x, \xi)| \leq C \sum_{i=1}^{N}\left|\xi_{i}\right|^{\frac{p_{i}}{p}}+b(x)
$$


If $\left\{u_{i_{k}}\right\} \subset L^{p_{i}}(\Omega)(1 \leq i \leq N)$ is bounded and $u_{i_{k}}$ convergent to $u_{i}$ in $\Omega_{0}$ for all bounded $\Omega_{0} \subset \Omega$, then for all $v \in L^{p^{\prime}}$, the following equality holds:

$$
\lim _{k \rightarrow \infty} \int_{\Omega} f\left(x, u_{1_{k}}, \ldots, u_{N_{k}}\right) v d x=\int_{\Omega} f\left(x, u_{1}, \ldots, u_{N}\right) v d x
$$

\section{Existence of solutions to abstract equations}

Let $G=A+B: X_{2} \times R^{+} \rightarrow X_{1}^{*}$. Assume:

(A1) There is a $C^{1}$ functional $F: X_{2} \rightarrow R^{1}$ such that

$$
\langle A u, L v\rangle=\langle-D F(u), v\rangle, \quad \forall u, v \in X
$$

(A2) Functional $F: X_{2} \rightarrow R^{1}$ is coercive, i.e.,

$$
F(u) \rightarrow \infty \quad \Leftrightarrow \quad\|u\|_{X_{2}} \rightarrow \infty
$$

(A3) $B$ satisfies

$$
|\langle B u, L v\rangle| \leq C_{1} F(u)+\frac{k}{2}\|v\|_{H_{1}}^{2}+g(t), \quad \forall u, v \in X,
$$

for $g \in L_{\text {loc }}^{1}(0, \infty)$.

Theorem 3.1 If G: $X_{2} \times R^{+} \rightarrow X_{1}^{*}$ is T-coercively weakly continuous, and

$$
\lim _{n \rightarrow \infty} \int_{0}^{t}\left|\left\langle G u_{n}-G u_{0}, L u_{n}-L u_{0}\right\rangle\right| d t+\lim _{n \rightarrow \infty}\left\|u_{n}-u_{0}\right\|_{H_{2}}^{2}=0,
$$

then for all $(\varphi, \psi) \in X_{2} \times H_{1}$, then the following assertions hold:

(1) If $G=A$ satisfies (A1) and (A2), then Eq. (2.4) has a global weak solution

$$
u \in W^{1, \infty}\left((0, \infty), H_{1}\right) \cap W^{1,2}\left((0, \infty), H_{2}\right) \cap L^{\infty}\left((0, \infty), X_{2}\right) .
$$

(2) If $G=A+B$ satisfies (A1)-(A3), then Eq. (2.4) has a global weak solution

$$
u \in W_{\text {loc }}^{1, \infty}\left((0, \infty), H_{1}\right) \cap W_{\text {loc }}^{1,2}\left((0, \infty), H_{2}\right) \cap L_{\text {loc }}^{\infty}\left((0, \infty), X_{2}\right) .
$$

(3) Furthermore, if $\mathcal{L}: X_{2} \rightarrow X_{1}$ is a symmetric sectorial operator, i.e., $\langle\mathcal{L} u, v\rangle=\langle u, \mathcal{L} v\rangle$, and $G=A+B$ satisfies

$$
|\langle G u, v\rangle| \leq C F(u)+\frac{1}{2}\|v\|_{H}^{2}+g(t)
$$

for $g \in L^{1}(0, T)$, then $u \in W_{\text {loc }}^{2,2}((0, \infty) ; H)$.

Proof Let $\left\{e_{k}\right\} \subset X$ be a common orthogonal basis of $H$ and $H_{3}$, satisfying (2.3). Set

$$
\left\{\begin{array}{l}
X_{n}=\left\{\sum_{i=1}^{n} \alpha_{i} e_{i} \mid \alpha_{i} \in R^{1}\right\} \\
\tilde{X}_{n}=\left\{\sum_{j=1}^{n} \beta_{j}(t) e_{j} \mid \beta_{j}(t) \in C^{2}[0, \infty)\right\}
\end{array}\right.
$$


Clearly, $L X_{n}=X_{n}, L \tilde{X}_{n}=\tilde{X}_{n}$.

By using the Galerkin method, there exists $u_{n} \in C^{2}\left([0, \infty), X_{n}\right)$ satisfying

$$
\left\{\begin{array}{l}
\left\langle\frac{d u_{n}}{d t}, v\right\rangle_{H}+k\left\langle\mathcal{L} u_{n}, v\right\rangle_{H}=\int_{0}^{t}\left\langle G\left(u_{n}\right), v\right\rangle d t+\left\langle\psi_{n}, v\right\rangle_{H}+k\left\langle\mathcal{L} \varphi_{n}, v\right\rangle_{H}, \\
u_{n}(0)=\varphi_{n}, \quad u_{n}^{\prime}(0)=\psi_{n},
\end{array}\right.
$$

for $\forall v \in X_{n}$, and

$$
\int_{0}^{t}\left[\left\langle\frac{d^{2} u_{n}}{d^{2} t}, v\right\rangle_{H}+k\left\langle\mathcal{L} \frac{d u_{n}}{d t}, v\right\rangle_{H}\right] d t=\int_{0}^{t}\left\langle G u_{n}, v\right\rangle d t
$$

for $\forall v \in \tilde{X}_{n}$.

Firstly, we consider $G=A$. Let $v=\frac{d}{d t} L u_{n}$ in (3.9). Taking into account (2.2) and (3.1), it follows that

$$
\begin{aligned}
0 & =\int_{0}^{t}\left[\frac{1}{2} \frac{d}{d t}\left\langle\frac{d u_{n}}{d t}, \frac{d u_{n}}{d t}\right\rangle_{H_{1}}+k\left\langle\frac{d u_{n}}{d t}, \frac{d u_{n}}{d t}\right\rangle_{H_{2}}+\left\langle D F\left(u_{n}\right), \frac{d u_{n}}{d t}\right\rangle\right] d t \\
& =\frac{1}{2}\left\|\frac{d u_{n}}{d t}\right\|_{H_{1}}^{2}-\frac{1}{2}\left\|\psi_{n}\right\|_{H_{1}}^{2}+k \int_{0}^{t}\left\|\frac{d u_{n}}{d t}\right\|_{H_{2}}^{2} d t+F\left(u_{n}\right)-F\left(\varphi_{n}\right) .
\end{aligned}
$$

We get

$$
\frac{1}{2}\left\|\frac{d u_{n}}{d t}\right\|_{H_{1}}^{2}+k \int_{0}^{t}\left\|\frac{d u_{n}}{d t}\right\|_{H_{2}}^{2} d t+F\left(u_{n}\right)=F\left(\varphi_{n}\right)+\frac{1}{2}\left\|\psi_{n}\right\|_{H_{1}}^{2}
$$

Let $\varphi \in H_{3}$. From (2.1) and (2.2), it is known that $\left\{e_{n}\right\}$ is an orthogonal basis of $H_{1}$. We find that $\varphi_{n} \rightarrow \varphi$ in $H_{3}$, and $\psi_{n} \rightarrow \psi$ in $H_{1}$. From that $H_{3} \subset X_{2}$ is an imbedding, it follows that

$$
\begin{cases}\varphi_{n} \rightarrow \varphi & \text { in } X_{2} \\ \psi_{n} \rightarrow \psi & \text { in } H_{1}\end{cases}
$$

From (3.2), (3.10), and (3.11), we obtain

$$
\left\{u_{n}\right\} \subset W^{1, \infty}\left((0, \infty), H_{1}\right) \cap W^{1,2}\left((0, \infty), H_{2}\right) \cap L^{\infty}\left((0, \infty), X_{2}\right) \quad \text { is bounded }
$$

Let

$$
\begin{cases}u_{n} \rightarrow{ }^{*} u_{0} & \text { in } W^{1, \infty}\left((0, \infty), H_{1}\right) \cap L^{\infty}\left((0, \infty), X_{2}\right), \\ u_{n} \rightarrow u_{0} & \text { in } W^{1,2}\left((0, \infty), H_{2}\right),\end{cases}
$$

which implies that $u_{n} \rightarrow u_{0}$ in $W^{1,2}((0, \infty), H)$ is uniformly weakly convergent from that $\mathrm{H}_{2} \subset \mathrm{H}$ is a compact imbedding.

If we have the following equality,

$$
\lim _{n \rightarrow \infty}\left[-\int_{0}^{t}\left|\left\langle G u_{n}-G u_{0}, L u_{n}-L u_{0}\right\rangle\right| d t+\frac{k}{2}\left\|u_{n}-u_{0}\right\|_{H_{2}}\right]=0,
$$


then $u_{0}$ is a weak solution of Eq. (2.4) in view of (3.8), (3.12), and $T$-coercively weakly continuous of $G$.

We will show (3.13) as follows. It follows that from (2.5)

$$
\begin{aligned}
\int_{0}^{t}\left\langle\frac{d}{d t} \mathcal{L} u_{n}-\frac{d}{d t} \mathcal{L} u_{0}, L u_{n}-L u_{0}\right\rangle_{H} d t & =\frac{1}{2} \int_{0}^{t} \frac{d}{d t}\left\langle u_{n}-u_{0}, u_{n}-u_{0}\right\rangle_{H_{2}} d t \\
& =\frac{1}{2}\left\|u_{n}(t)-u_{0}(t)\right\|_{H_{2}}^{2}-\frac{1}{2}\left\|\varphi_{n}-\varphi\right\|_{H_{2}}^{2} .
\end{aligned}
$$

Taking into account (2.2), (2.5) and (3.9), we get that

$$
\begin{aligned}
- & \int_{0}^{t}\left\langle G u_{n}-G u_{0}, L u_{n}-L u_{0}\right\rangle d t+\frac{k}{2}\left\|u_{n}-u_{0}\right\|_{H_{2}} \\
= & \int_{0}^{t}\left[\left\langle G u_{0}-G u_{n}, L u_{n}-L u_{0}\right\rangle\right. \\
& \left.+k\left\langle\frac{d}{d t} \mathcal{L} u_{n}-\frac{d}{d t} \mathcal{L} u_{0}, L u_{n}-L u_{0}\right\rangle_{H}\right] d t+\frac{k}{2}\left\|\varphi_{n}-\varphi\right\|_{H_{2}}^{2} \\
= & \int_{0}^{t}\left[\left\langle G u_{0}, L u_{n}-L u_{0}\right\rangle+\left\langle G u_{n}, L u_{0}\right\rangle-k\left\langle\frac{d u_{n}}{d t}, u_{0}\right\rangle_{H_{2}}-k\left\langle\frac{d}{d t} u_{0}, u_{n}-u_{0}\right\rangle_{H_{2}}\right. \\
& \left.+\left\langle\frac{d u_{n}}{d t}, \frac{d u_{n}}{d t}\right\rangle_{H_{1}}\right] d t-\left\langle\frac{d u_{n}}{d t}, u_{n}\right\rangle_{H_{1}}+\left\langle\psi_{n}, \varphi_{n}\right\rangle_{H_{1}}+\frac{k}{2}\left\|\varphi_{n}-\varphi\right\|_{H_{2}}^{2} .
\end{aligned}
$$

From (2.1) and (3.12), we have

$$
\begin{aligned}
& \lim _{n \rightarrow \infty}\left\|\varphi_{n}-\varphi\right\|_{H_{2}}=0, \\
& \lim _{n \rightarrow \infty} \int_{0}^{t}\left\langle G u_{0}, L u_{n}-L u_{0}\right\rangle d t=0, \\
& \lim _{n \rightarrow \infty} \int_{0}^{t}\left\langle\frac{d}{d t} u_{0}, u_{n}-u_{0}\right\rangle_{H_{2}} d t=0 .
\end{aligned}
$$

Then we get

$$
\begin{aligned}
\lim _{n \rightarrow \infty} & -\int_{0}^{t}\left\langle G u_{n}-G u_{0}, L u_{n}-L u_{0}\right\rangle d t+\frac{k}{2} \lim _{n \rightarrow \infty}\left\|u_{n}-u_{0}\right\|_{H_{2}}^{2} \\
= & \lim _{n \rightarrow \infty} \int_{0}^{t}\left[\left\langle G u_{n}, L u_{0}\right\rangle-k\left\langle\frac{d u_{n}}{d t}, u_{0}\right\rangle_{H_{2}}+\left\|\frac{d u_{n}}{d t}\right\|_{H_{1}}^{2}\right] d t \\
& -\lim _{n \rightarrow \infty}\left\langle\frac{d u_{n}}{d t}, u_{n}\right\rangle_{H_{1}}+\langle\psi, \varphi\rangle_{H_{1}} .
\end{aligned}
$$

In view of (3.9), (3.12), we obtain for all $v \in \bigcup_{n=1}^{\infty} \tilde{X}_{n}$

$$
\begin{aligned}
\lim _{n \rightarrow \infty} \int_{0}^{t}\left\langle G u_{n}, L v\right\rangle d t= & \int_{0}^{t}\left[k\left\langle\frac{d u_{0}}{d t}, v\right\rangle_{H_{2}}-\left\langle\frac{d u_{0}}{d t}, \frac{d v}{d t}\right\rangle_{H_{1}}\right] d t \\
& +\left\langle\frac{d u_{0}}{d t}, v\right\rangle_{H_{1}}-\langle\psi, v(0)\rangle_{H_{1}} .
\end{aligned}
$$


Since $\bigcup_{n=1}^{\infty} \tilde{X}_{n}$ is dense in $W^{1,2}\left((0, T), H_{2}\right) \cap L^{p}\left((0, T), X_{2}\right), \forall p<\infty$, (3.15) holds for all $v \in W^{1,2}\left((0, T), H_{2}\right) \cap L^{p}\left((0, T), X_{2}\right)$. Thus, we have

$$
\begin{aligned}
\lim _{n \rightarrow \infty} \int_{0}^{t}\left\langle G u_{n}, L u_{0}\right\rangle d t= & \int_{0}^{t}\left[k\left\langle\frac{d u_{0}}{d t}, u_{0}\right\rangle_{H_{2}}-\left\|\frac{d u_{0}}{d t}\right\|_{H_{1}}^{2}\right] d t \\
& +\left\langle\frac{d u_{0}}{d t}, u_{0}\right\rangle_{H_{1}}-\langle\psi, \varphi\rangle_{H_{1}} .
\end{aligned}
$$

From (3.12) and $H_{2} \subset H_{1}$ is compact imbedding, it follows that

$$
\begin{aligned}
& \lim _{n \rightarrow \infty} \int_{0}^{t}\left\|\frac{d u_{n}}{d t}\right\|_{H_{1}}^{2} d t=\int_{0}^{t}\left\|\frac{d u_{0}}{d t}\right\|_{H_{1}}^{2} d t, \\
& \lim _{n \rightarrow \infty}\left\langle\frac{d u_{n}}{d t}, u_{n}\right\rangle_{H_{1}}=\left\langle\frac{d u_{0}}{d t}, u_{0}\right\rangle_{H_{1}}, \quad \text { a.e. } t \geq 0 .
\end{aligned}
$$

Clearly,

$$
\lim _{n \rightarrow \infty} \int_{0}^{t}\left\langle\frac{d u_{n}}{d t}, u_{n}\right\rangle_{H_{1}} d t=\int_{0}^{t}\left\langle\frac{d u_{0}}{d t}, u_{0}\right\rangle_{H_{1}} d t
$$

Then (3.13) follows from (3.14)-(3.16), which imply assertion (1).

Secondly, we consider $G=A+B$. Let $v=\frac{d}{d t} L u_{n}$ in (3.9). In view of (2.2) and (3.1), it follows that

$$
\frac{1}{2}\left\|\frac{d u_{n}}{d t}\right\|_{H_{1}}^{2}+k \int_{0}^{t}\left\|\frac{d u_{n}}{d t}\right\|_{H_{2}}^{2} d t+F\left(u_{n}\right)=\int_{0}^{t}\left\langle B\left(u_{n}\right), \frac{d}{d t} L u_{n}\right\rangle d t+F\left(\varphi_{n}\right)+\frac{1}{2}\left\|\psi_{n}\right\|_{H_{1}}^{2} .
$$

From (3.3), we have

$$
\frac{1}{2}\left\|\frac{d u_{n}}{d t}\right\|_{H_{1}}^{2}+F\left(u_{n}\right)+k \int_{0}^{t}\left\|\frac{d u_{n}}{d t}\right\|_{H_{2}}^{2} d t \leq C \int_{0}^{t}\left[F\left(u_{n}\right)+\frac{1}{2}\left\|\frac{d u_{n}}{d t}\right\|_{H_{1}}^{2}\right] d t+f(t)
$$

where $f(t)=\int_{0}^{t} g(\tau) d \tau+\frac{1}{2}\|\psi\|_{H_{1}}^{2}+\sup _{n} F\left(\varphi_{n}\right)$.

By using the Gronwall inequality, it follows that

$$
\frac{1}{2}\left\|\frac{d u_{n}}{d t}\right\|_{H_{1}}^{2}+F\left(u_{n}\right) \leq f(0) e^{C t}+\int_{0}^{t} f(\tau) e^{C(t-\tau)} d \tau,
$$

which implies that for all $0<T<\infty$,

$$
\left\{u_{n}\right\} \subset W^{1, \infty}\left((0, T), H_{1}\right) \cap L^{\infty}\left((0, T), X_{2}\right) \quad \text { is bounded. }
$$

From (3.17) and (3.18), it follows that

$$
\left\{u_{n}\right\} \subset W^{1,2}\left((0, T), H_{2}\right) \quad \text { is bounded. }
$$

Let

$$
\begin{cases}u_{n} \rightarrow{ }^{*} u_{0} & \text { in } W^{1, \infty}\left((0, T), H_{1}\right) \cap L^{\infty}\left((0, T), X_{2}\right), \\ u_{n} \rightarrow u_{0} & \text { in } W^{1,2}\left((0, T), H_{2}\right),\end{cases}
$$


which implies that $u_{n} \rightarrow u_{0}$ in $W^{1,2}((0, T), H)$ is uniformly weakly convergent from that $\mathrm{H}_{2} \subset \mathrm{H}$ is an compact imbedding.

The left proof is same as assertion (1).

Lastly, assume (3.6) holds. Let $v=\frac{d^{2} u_{n}}{d^{2} t}$ in (3.9). It follows that

$$
\begin{aligned}
& \int_{0}^{t}\left\langle\frac{d^{2} u_{n}}{d t^{2}}, \frac{d^{2} u_{n}}{d t^{2}}\right\rangle_{H} d t+\frac{k}{2}\left\|\frac{d u_{n}}{d t}\right\|_{H_{1}}^{2} \\
& \quad \leq \frac{k}{2}\left\|\psi_{n}\right\|_{H}^{2}+\int_{0}^{t}\left[\frac{1}{2}\left\|\frac{d^{2} u_{n}}{d t^{2}}\right\|_{H}^{2}+C F\left(u_{n}\right)+g(\tau)\right] d \tau .
\end{aligned}
$$

From (3.18), the above inequality implies

$$
\int_{0}^{t}\left\|\frac{d^{2} u_{n}}{d t^{2}}\right\|_{H}^{2} d \tau \leq C \quad(C>0 \text { is constant })
$$

We see that for all $0<T<\infty,\left\{u_{n}\right\} \subset W^{2,2}((0, T), H)$ is bounded. Thus, $u \in W^{2,2}((0, T), H)$.

\section{Main result}

We consider the strongly damped quasilinear wave equations (1.1). We give the following assumption for (1.1). There exists an $C^{1}$ function $F(x, \zeta)$, where $\zeta=\left\{\zeta_{\alpha}|| \alpha \mid \leq m\right\}, \zeta_{\alpha}$ corresponds to $D^{\alpha} u$ such that

$$
\begin{aligned}
& A_{\alpha}(x, \zeta)=\frac{\partial}{\partial \zeta_{\alpha}} F(x, \zeta), \\
& F(x, \zeta) \geq C_{1} \sum_{|\beta|=m}\left|\zeta_{\beta}\right|^{p}-C_{2}, \quad p \geq 2, \\
& \sum_{|\beta|=m}\left[A_{\beta}\left(x, \xi, \eta_{1}\right)-A_{\beta}\left(x, \xi, \eta_{2}\right)\right]\left(\eta_{1 \beta}-\eta_{2 \beta}\right) \geq \lambda\left|\eta_{1}-\eta_{2}\right|^{2},
\end{aligned}
$$

where $\lambda>0, \eta=\left\{\eta_{\beta}|| \beta \mid=m\right\}, \xi=\left\{\xi_{\alpha}|| \alpha \mid \leq m-1\right\}$,

$$
\begin{aligned}
& \left|A_{\alpha}(x, \zeta)\right| \leq C\left(\sum_{|\alpha| \leq m}\left|\zeta_{\alpha}\right|^{p-1}+1\right) \\
& |g(x, \zeta)| \leq C\left(\sum_{|\beta| \leq m}\left|\zeta_{\beta}\right|^{\frac{p}{2}}+1\right) .
\end{aligned}
$$

Definition 4.1 We say $u \in W_{\text {loc }}^{1,2}\left((0, \infty), L^{2}(\Omega)\right) \cap L_{\text {loc }}^{\infty}\left((0, \infty), W_{0}^{m, p}(\Omega)\right)$ is the weak solution of (1.1), if $u(0)=\varphi$, and for $\forall v \in C_{0}^{\infty}(\Omega)$, the following equality holds:

$$
\begin{aligned}
\int_{\Omega} & \frac{\partial u}{\partial t} v d x+k \int_{\Omega} \nabla u \nabla v d x \\
= & -\int_{0}^{t} \int_{\Omega} \sum_{|\alpha| \leq m} D^{\alpha} A_{\alpha}\left(x, u, \ldots, D^{m} u\right) D^{\alpha} v d x d \tau \\
& +\int_{0}^{t} \int_{\Omega} g\left(x, u, \ldots, D^{m} u\right) v d x d t+\int_{\Omega} \psi v d x+k \int_{\Omega} \nabla \varphi \nabla v d x .
\end{aligned}
$$


Theorem 4.2 Under conditions (4.1)-(4.5), for $(\varphi, \psi) \in W_{0}^{m, p}(\Omega) \times L^{p}(\Omega)$, there exists a global weak solution for (1.1)

$$
\begin{aligned}
& u \in L_{\mathrm{loc}}^{\infty}\left((0, \infty), W_{0}^{m, p}(\Omega)\right), \\
& u_{t} \in L_{\mathrm{loc}}^{\infty}\left((0, \infty), L^{2}(\Omega)\right) \cap L_{\mathrm{loc}}^{2}\left((0, \infty), H_{0}^{1}(\Omega)\right) .
\end{aligned}
$$

Proof We introduce spatial sequences

$$
\begin{aligned}
& X=C_{0}^{\infty}(\Omega), \quad X_{1}=X_{2}=W_{0}^{m, p}(\Omega), \\
& H=H_{1}=L^{2}(\Omega), \quad H_{2}=H_{0}^{1}(\Omega), \\
& L=i d: X \rightarrow X_{1}, \quad \mathcal{L}=-\Delta u .
\end{aligned}
$$

Define map $G=A+B: X_{2} \rightarrow X_{1}^{*}$ by

$$
\begin{aligned}
& \langle A u, v\rangle=-\int_{\Omega} \sum_{|\alpha| \leq m} A_{\alpha}\left(x, u, \ldots, D^{m} u\right) D^{\alpha} v d x, \\
& \langle B u, v\rangle=\int_{\Omega} g\left(x, u, \ldots, D^{m} u\right) v d x .
\end{aligned}
$$

We show that $G=A+B: X_{2} \rightarrow X_{1}^{*}$ is $T$-coercively weakly continuous. Let $\left\{u_{n}\right\} \subset$ $L^{\infty}\left(0, T, W^{2, p}(\Omega) \cap W_{0}^{1, p}(\Omega)\right)$ satisfying $(2.7)$ and

$$
\begin{aligned}
& \lim _{n \rightarrow \infty} \int_{0}^{T} \int_{\Omega}\left[\left(\sum_{|\alpha| \leq m} A_{\alpha}\left(x, u_{n}, \ldots, D^{m} u_{n}\right)-\sum_{|\alpha| \leq m} A_{\alpha}\left(x, u_{0}, \ldots, D^{m} u_{0}\right)\right)\left(D^{\alpha} u_{n}-D^{\alpha} u_{0}\right)\right. \\
& \left.\quad+\left(g\left(x, u_{n}, \ldots, D^{m} u_{n}\right)-g\left(x, u_{0}, \ldots, D^{m} u_{0}\right)\right)\left(u_{n}-u_{0}\right)\right] d x d t=0
\end{aligned}
$$

We need prove that

$$
\begin{gathered}
\lim _{n \rightarrow \infty} \int_{0}^{T} \int_{\Omega}\left[\sum_{|\alpha| \leq m} A_{\alpha}\left(x, u_{n}, \ldots, D^{m} u_{n}\right)+g\left(x, u_{n}, \ldots, D^{m} u_{n}\right)\right] v d x d t \\
\quad=\int_{0}^{T} \int_{\Omega}\left[\sum_{|\alpha| \leq m} A_{\alpha}\left(x, u_{0}, \ldots, D^{m} u_{0}\right)+g\left(x, u_{0}, \ldots, D^{m} u_{0}\right)\right] v d x d t
\end{gathered}
$$

From (2.7) and Lemma 2.4, we obtain

$$
u_{n} \rightarrow u_{0}, \quad D u_{n} \rightarrow D u_{0}, \ldots, \quad D^{m-1} u_{n} \rightarrow D^{m-1} u_{0}, \quad \text { in } L^{2}((0, T) \times \Omega) .
$$

We have the deformation

$$
\begin{aligned}
& \int_{0}^{T} \int_{\Omega}\left[\left(\sum_{|\alpha| \leq m} A_{\alpha}\left(x, u_{n}, \ldots, D^{m} u_{n}\right)-\sum_{|\alpha| \leq m} A_{\alpha}\left(x, u_{0}, \ldots, D^{m} u_{0}\right)\right)\left(D^{\alpha} u_{n}-D^{\alpha} u_{0}\right)\right] d x d t \\
& \quad+\int_{0}^{T} \int_{\Omega}\left[g\left(x, u_{n}, \ldots, D^{m} u_{n}\right)-g\left(x, u_{0}, \ldots, D^{m} u_{0}\right)\right]\left(u_{n}-u_{0}\right) d x d t
\end{aligned}
$$




$$
\begin{aligned}
= & \int_{0}^{T} \int_{\Omega}\left[\left(\sum_{|\alpha| \leq m} A_{\alpha}\left(x, u_{n}, \ldots, D^{m-1} u_{n}, D^{m} u_{0}\right)\right.\right. \\
& \left.\left.-\sum_{|\alpha| \leq m} A_{\alpha}\left(x, u_{0}, \ldots, D^{m-1} u_{0}, D^{m} u_{0}\right)\right)\left(D^{\alpha} u_{n}-D^{\alpha} u_{0}\right)\right] d x d t \\
& +\int_{0}^{T} \int_{\Omega}\left[\left(\sum_{|\alpha| \leq m} A_{\alpha}\left(x, u_{n}, \ldots, D^{m-1} u_{n}, D^{m} u_{n}\right)\right.\right. \\
& \left.\left.-\sum_{|\alpha| \leq m} A_{\alpha}\left(x, u_{n}, \ldots, D^{m-1} u_{n}, D^{m} u_{0}\right)\right)\left(D^{\alpha} u_{n}-D^{\alpha} u_{0}\right)\right] d x d t \\
& +\int_{0}^{T} \int_{\Omega}\left[g\left(x, u_{n}, \ldots, D^{m} u_{n}\right)-g\left(x, u_{0}, \ldots, D^{m} u_{0}\right)\right]\left(u_{n}-u_{0}\right) d x d t .
\end{aligned}
$$

From (4.9), (4.4), (4.5), and Lemma 2.5, we have

$$
\begin{aligned}
& \lim _{n \rightarrow \infty} \int_{0}^{T} \int_{\Omega}\left[g\left(x, u_{n}, \ldots, D^{m} u_{n}\right)-g\left(x, u_{0}, \ldots, D^{m} u_{0}\right)\right]\left(u_{n}-u_{0}\right) d x d t=0 \\
& \int_{0}^{T} \int_{\Omega}\left[\sum_{|\alpha| \leq m} A_{\alpha}\left(x, u_{n}, \ldots, D^{m-1} u_{n}, D^{m} u_{0}\right)\right. \\
& \left.\quad-\sum_{|\alpha| \leq m} A_{\alpha}\left(x, u_{0}, \ldots, D^{m-1} u_{0}, D^{m} u_{0}\right)\right]\left(D^{\alpha} u_{n}-D^{\alpha} u_{0}\right) d x d t=0 .
\end{aligned}
$$

From (4.7), (4.3), (4.10)-(4.12), it follows that

$$
\begin{aligned}
0= & \int_{0}^{T} \int_{\Omega}\left[\left(\sum_{|\alpha| \leq m} A_{\alpha}\left(x, u_{n}, \ldots, D^{m-1} u_{n}, D^{m} u_{n}\right)\right.\right. \\
& \left.\left.-\sum_{|\alpha| \leq m} A_{\alpha}\left(x, u_{n}, \ldots, D^{m-1} u_{n}, D^{m} u_{0}\right)\right)\left(D^{\alpha} u_{n}-D^{\alpha} u_{0}\right)\right] d x d t \\
\geq & \lambda \int_{0}^{T} \int_{\Omega}\left|D^{m} u_{n}-D^{m} u_{0}\right|^{2} d x d t .
\end{aligned}
$$

Since $\lambda>0$, we have

$$
\lim _{n \rightarrow \infty} \int_{0}^{T} \int_{\Omega}\left|D^{m} u_{n}-D^{m} u_{0}\right|^{2} d x d t=0
$$

From (4.9), (4.13), (4.4), (4.5), and Lemma 2.5, we get (4.8). Hence, $G=A+B: X_{2} \rightarrow X_{1}^{*}$ is $T$-coercively weakly continuous.

From (4.1) and (4.2), we get

$$
\begin{aligned}
& \langle A u, L u\rangle=-\langle D F(x, \zeta), v\rangle, \\
& F(x, u) \rightarrow \infty \quad \Leftrightarrow \quad\|u\|_{X_{2}} \rightarrow \infty,
\end{aligned}
$$

which imply conditions (A1), (A2) of Theorem 3.1. 
We will show (3.3) as follows. It follows that

$$
\begin{aligned}
|\langle B u, L v\rangle| & =\int_{\Omega}\left|g\left(x, u, \ldots, D^{m} u\right)\right||v| d x \\
& \leq \frac{k}{2} \int_{\Omega}|v|^{2} d x+\frac{2}{k} \int_{\Omega}\left|g\left(x, u, \ldots, D^{m} u\right)\right|^{2} d x \\
& \leq \frac{k}{2}\|v\|_{H_{2}}^{2}+C \int_{\Omega}\left[\sum_{|\alpha| \leq m}|\zeta|^{\frac{p}{2}}+1\right]^{2} d x \\
& \leq \frac{k}{2}\|v\|_{H_{2}}^{2}+C F(u)+C,
\end{aligned}
$$

which implies condition (A3) of Lemma 2.4. From Lemma 2.4, Eq. (1.1) has a solution

$$
\begin{aligned}
& u \in L_{\mathrm{loc}}^{\infty}\left((0, \infty), W_{0}^{m, p}(\Omega)\right), \\
& u_{t} \in L_{\mathrm{loc}}^{\infty}\left((0, \infty), L^{2}(\Omega)\right) \cap L_{\mathrm{loc}}^{2}\left((0, \infty), H_{0}^{1}(\Omega)\right),
\end{aligned}
$$

satisfying

$$
\begin{aligned}
\int_{\Omega} & \frac{\partial u}{\partial t} v d x+k \int_{\Omega} \nabla u \nabla v d x \\
= & -\int_{0}^{t} \int_{\Omega} \sum_{|\alpha| \leq m} D^{\alpha} A_{\alpha}\left(x, u, \ldots, D^{m} u\right) D^{\alpha} v d x d \tau \\
& +\int_{0}^{t} \int_{\Omega} g\left(x, u, \ldots, D^{m} u\right) v d x d t+\int_{\Omega} \psi v d x+k \int_{\Omega} \nabla \varphi \nabla v d x .
\end{aligned}
$$

\section{Competing interests}

The authors declare that they have no competing interests.

\section{Authors' contributions}

All authors typed, read and approved the final manuscript.

\section{Author details}

${ }^{1}$ College of Mathematics and Software Science, Sichuan Normal University, Chengdu, Sichuan 610066, China. ${ }^{2}$ College of Mathematics, Sichuan University, Chengdu, Sichuan 610041, China.

\section{Acknowledgements}

The authors are very grateful to the anonymous referees whose careful reading of the manuscript and valuable comments enhanced the presentation of the manuscript. Foundation item: the National Natural Science Foundation of China (No. 11071177), the NSF of Sichuan Science and Technology Department of China (No. 2010JY0057), and the NSF of the Sichuan Education Department of China (No. 11ZA102).

Received: 23 May 2012 Accepted: 23 July 2012 Published: 8 August 2012

\section{References}

1. Webb, GF: Existence and asymptotic behavior for a strongly damped nonlinear wave equation. Can. J. Math. 32 , 631-643 (1980)

2. Carvalho, AN, Cholewa, JW: Local well posedness for strongly damped wave equations with critical nonlinearities. Bull. Aust. Math. Soc. 66, 443-463 (2002)

3. Carvalho, AN, Cholewa, JW: Attractors for strongly damped wave equations with critical nonlinearities. Pac. J. Math. 207, 287-310 (2002)

4. Cholewa, JW, Dlotko, T: Strongly damped wave equation in uniform spaces. Nonlinear Anal. 64, 174-187 (2006)

5. Ghidaglia, JM, Marzocchi, A: Longtime behaviour of strongly damped wave equations, global attractors and their dimensions. SIAM J. Math. Anal. 22, 879-895 (1991)

6. Massatt, P: Longtime behaviour of strongly damped nonlinear wave equations. J. Differ. Equ. 48, 334-349 (1983)

7. Temam, R: Infinite Dimensional Dynamical Systems in Mechanics and Physics, 2nd edn. Springer, New York (1997) 
8. Zhou, S: Global attractor for strongly damped nonlinear wave equations. Funct. Differ. Equ. 6, 451-470 (1999)

9. Zhou, S, Fan, X: Kernel sections for non-autonomous strongly damped wave equations. J. Math. Anal. Appl. 275 850-869 (2002)

10. Fan, X, Zhou, S: The inflated attractors of non-autonomous strongly damped wave equations. Acta Math. Appl. Sinica (Engl. Ser.) 20(4), 547-556 (2004)

11. Li, H, Zhou, S: Global periodic attractor for strongly damped and driven wave equations. Acta Math. Appl. Sinica (Engl. Ser.) 22(1), 75-80 (2006)

12. Belleri, $V$, Pata, V: Attractors for semilinear strongly damped wave equation on $R^{3}$. Discrete Contin. Dyn. Syst. 7, 719-735 (2001)

13. Carvalho, AN, Cholewa, JW: Attractors for strongly damped wave equations with critical nonlinearities. Pac. J. Math. 207, 287-310 (2002)

14. Ghidaglia, JM, Marzocchi, A: Longtime behaviour of strongly damped wave equations, global attractors and their dimension. SIAM J. Math. Anal. 22, 879-895 (1991)

15. Massat, P: Limiting behavior for strongly damped nonlinear wave equations. J. Differ. Equ. 48, 334-349 (1983)

16. Yang, H, Liu, F: Boundary value problem for quasilinear wave equation. J. Math. Study 32(2), 156-160 (1999)

17. Greenberg, JM, Li, T: The effect of boundary value problem for the quasilinear wave equation. J. Differ. Equ. 52, 66-75 (1984)

18. Li, T: Global Classical Solutions for Quasilinear Hyperbolic Systems. Research Appl. Math., vol. 32. Masson/Wiley, New York (1994)

19. Li, T, Qin, T: Global smooth solutions for a class of quasilinear hyperbolic systems with dissipation. Chin. Ann. Math., Ser. B 6, 199-210 (1985)

20. Li, T, Yu, W: BVPs for Quasilinear Hyperbolic Systems. Duke University Mathematics Series, vol. 5. Duke University Press, Durham (1985)

21. Alinhac, S: The null condition for quasilinear wave equations in two space dimensions I. Invent. Math. 145, 597-618 (2001)

22. Alinhac, S: The null condition for quasilinear wave equations in two space dimensions II. Am. J. Math. 123(6), 1071-1101 (2001)

23. Zhang, W: Cauchy problem for quasilinear hyperbolic systems with higher order dissipative terms. Acta Math. Appl. Sinica (Engl. Ser.) 19(1), 71-82 (2003)

24. Metcalfe, J, Sogge, CD: Hyperbolic trapped rays and global existence of quasilinear wave equations. Invent. Math. $159,75-117(2005)$

25. Yin, $\mathrm{H}$ : The asymptotic behaviour of solutions for a class of quasilinear wave equations with cubic nonlinearity in two space dimensions. Acta Math. Appl. Sin. 16(3), 299-312 (2000)

26. Weidemaier, P: Existence of regular solutions for a quasilinear wave equation with the third boundary condition. Math. Z. 191, 449-465 (1985)

27. Chen, $G$, Yang, Z, Zhao, Z: Initial value problems and first boundary problems for a class of quasilinear wave equations. Acta Math. Appl. Sin. 9(4), 289-301 (1993)

28. Grille, A: On the numerical solution of quasilinear wave equation with strong dissipative term. Appl. Math. Mech. 25(7), 806-811 (2004)

29. Chen, F, Guo, B, Wang, P: Long time behavior of strongly damped nonlinear wave equations. J. Differ. Equ. 147 231-241 (1998)

30. Ma, T: Theories and Methods for Partial Differential Equations. Science Press, Beijing (2011) (Chinese)

doi:10.1186/1687-1847-2012-139

Cite this article as: Luo et al.: Existence of solutions to strongly damped quasilinear wave equations. Advances in Difference Equations 2012 2012:139.

\section{Submit your manuscript to a SpringerOpen ${ }^{\ominus}$ journal and benefit from:}

- Convenient online submission

Rigorous peer review

- Immediate publication on acceptance

- Open access: articles freely available online

- High visibility within the field

- Retaining the copyright to your article 